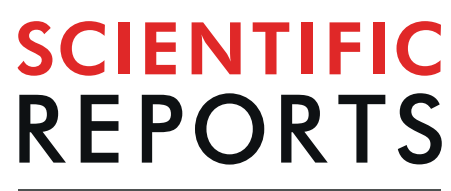

natureresearch

\title{
OPEN Active control on switchable waveguide of elastic wave metamaterials with the 3D printing technology
}

\author{
Guan-Hua Li ${ }^{1}$, Yi-Ze Wang ${ }^{2 *}$ \& Yue-Sheng Wang ${ }^{1,2}$ \\ Propagation of elastic waves along a direction has special interests in practical applications. These \\ concerns generate the design of an elastic wave metamaterial with electrically switchable properties, \\ which is studied in this work. The structure contains a T-shaped waveguide in a plate with the 3D printing \\ technology; and the active control system is used to tune the propagation direction of the flexural \\ wave. The piezoelectric patches which are connected by the negative capacitance circuits are applied to \\ behave as the active control system. The finite element simulation is performed to give the theoretical \\ prediction of the switchable waveguide and the tunable equivalent parameters are achieved by the \\ electrical circuits. The active control experiments are finally carried out to support the numerical design.
}

During the past decades, phononic crystals and elastic wave metamaterials which have the periodic characteristics have received lots of attention ${ }^{1-8}$. This is mainly because that these periodic systems have superior properties, such as the wave band gaps ${ }^{9-12}$, negative refraction ${ }^{13-16}$, acoustic cloaks ${ }^{17-19}$, etc. These behaviors can be used to tune the wave propagation. Acoustic couplers ${ }^{20}$, sensors ${ }^{21}$ and waveguides ${ }^{22}$ are several typical applications of phononic crystals and elastic wave metamaterials.

Another important characteristic of phononic crystals and elastic wave metamaterials is the defect state. It's well known that if there are some defects in these periodic structures, the elastic wave will be localized near the defect (for the point defect) or propagate along the defects (for the line defects). So the propagation direction and some other properties of elastic waves can be controlled by designing different waveguides. For instance, Rostami-Dogolsara et al. ${ }^{23}$ presented the switchable acoustic demultiplexers based on the fluid-fluid phononic crystals. Zhang et al. ${ }^{24}$ used the supercell plane wave method to investigate defect modes by introducing the bend-shaped linear defects. Wang et al. ${ }^{25,26}$ discussed wave guides with the reconfigurable and coupled-resonator properties. Yao et al..$^{27}$ presented the flexural waves in the phononic crystal plate with linear defects to show the guide behaviors.

In recent years, special attention has been focused on the active control effects on phononic crystals and elastic wave metamaterials, which can show many interesting results ${ }^{28-32}$. In order to derive the broadband control of elastic waves in a flexible plate, Tateo et al. ${ }^{33}$ presented an experimental investigation about a periodic array of shunted piezoelectric patches with the negative capacitance. Casadei et al. ${ }^{34}$ discussed about the experimental demonstration of a tunable acoustic waveguide which was implemented on a phononic plate. By connecting gradient negative capacitance circuits to an array of piezoelectric patches, Chen et al. ${ }^{35}$ designed a metamaterial-based sensing system with the gradient bending stiffness. They demonstrated that the proposed system can achieve more than two orders of magnitude amplification of flexural wave signals to overcome the detection limit. In this work, we propose an elastic wave metamaterial with a T-shaped switchable waveguide by the active control system. With the electrical tunability, the switchable properties of the elastic wave are realized.

On the other hand, compared with the traditional materials, phononic crystals and elastic wave metamaterials have unique band gap characteristics. At present, they have shown broad prospects in waveguides, filters, new vibration and noise reduction materials and other devices. Phononic crystals and elastic wave metamaterials have become the hot research topic in many fields, such as engineering mechanics, physics, mechanical engineering, etc. The research in this field has attracted extensive attention and has broad development prospects. 


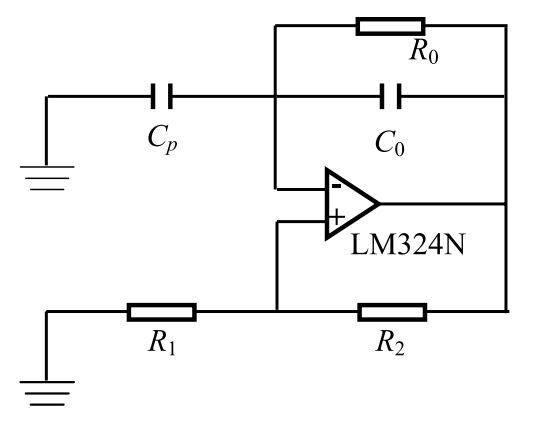

(a)

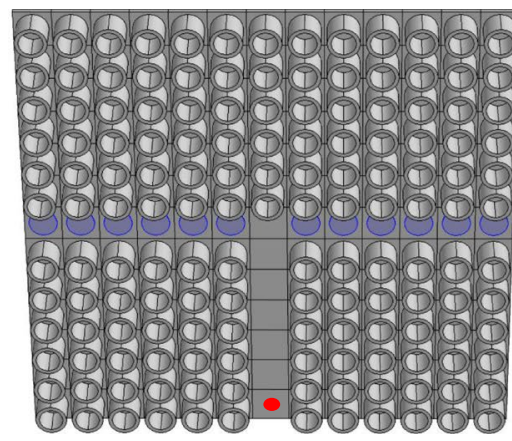

(b)

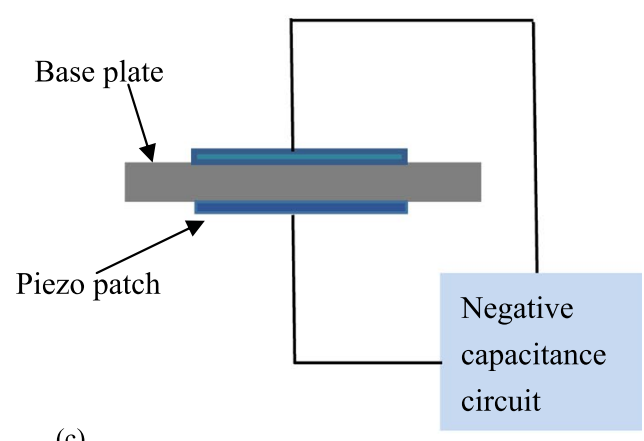

(c)

Figure 1. (a) Negative capacitance circuit. Finite element model: (b) the elastic metamaterial plate with a T-shape waveguide; and (c) the active unit cell with a piezoelectric patch attached by the negative capacitance circuit.

Nowadays, the 3D printing technology has gradually replaced the traditional manufacturing technology. It's mainly due to that the 3D printing technology can make most structural shapes with the precise size at different scales. As the structure becomes more complex by this method, the manufacturing cost will not increase. The 3D printing technology offers the opportunity for the traditional design and control of periodically patterned structures. And even it can realize some special characteristics, e.g. the negative stiffness and Poisson's ratio ${ }^{36}$. So based on the $3 \mathrm{D}$ printing technology, special phononic crystals and elastic wave metamaterials would be achieved.

\section{Results and Discussions}

In this section, the active control system is bonded on an elastic wave metamaterial plate which consists of the piezoelectric patches and negative capacitance circuits. Phononic crystals and elastic wave metamaterials have band gaps, in which elastic waves cannot propagate. When the elastic metamaterials have the defect like the point and line cases, the elastic wave will be limited to the point defect or propagate along the line defect. Phononic crystals and elastic wave metamaterials are the artificial structures with the periodic elastic constants and densities. Negative capacitance circuits can change the inherent elastic modules, which results in tuning the band gap structure. Then the elastic waves in specific frequencies can be controlled to propagate along the direction as we design. Then the propagation direction of elastic waves will be changed actively. Based on the finite element method, switchable properties of elastic waves in the metamaterial plate will be presented and the validity of numerical results will be verified by the experiment.

Negative capacitance circuit. Assume that the polarization direction is along the $z$-axis, and the $x, y$ and $z$ directions are described as 1,2 and 3, respectively. The piezoelectric equation can be expressed as ${ }^{37}$

$$
\begin{aligned}
& S_{1}=s_{11}^{E} T_{1}+d_{31} E_{3} \\
& D_{3}=d_{31} T_{1}+\varepsilon_{33}^{T} E_{3}
\end{aligned}
$$

where $S_{1}$ and $T_{1}$ denote the strain and stress along the $x$ direction; $S_{11}^{E}$ is the compliance coefficient of the piezoelectric material; $d_{31}$ and $\varepsilon_{33}^{T}$ are the piezoelectric and dielectric constants; $E_{3}$ is the electric field intensity along the $z$ direction; and $D_{3}$ is the electric displacement.

Substituting the expressions of the electric displacement and electric field into Eq. (1), the relation between the strain and stress becomes ${ }^{38}$ 

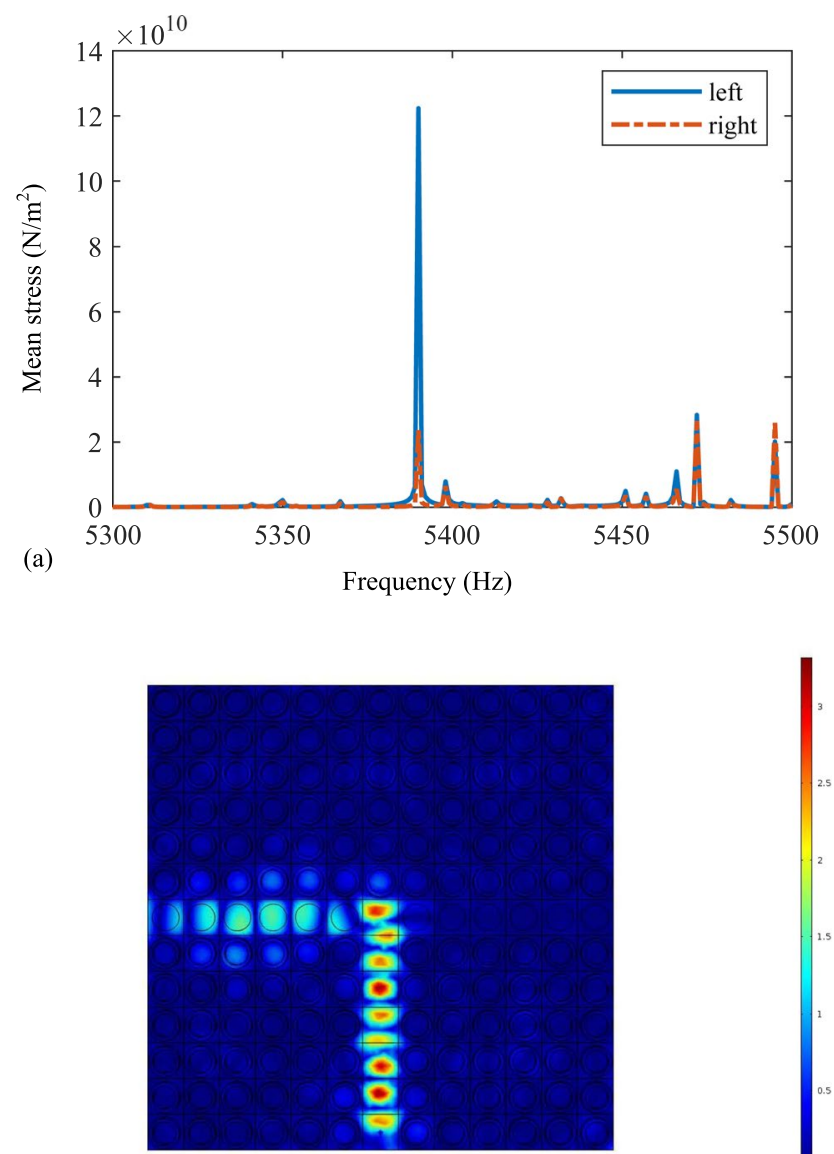

(b)

Figure 2. The switchable waveguide when the negative capacitance circuits are connected by the left side of the horizontal channel: (a) the mean stress of the left and right from $5300 \mathrm{~Hz}$ to $5500 \mathrm{~Hz}$, and (b) the wave mode at $5390 \mathrm{~Hz}$.

$$
S_{1}=\left(s_{11}^{E}-\frac{s Z d_{31}^{2} A_{s} h_{p}^{-1}}{1+s Z C_{p}}\right) T_{1}
$$

where $s$ is Laplacian operator; $Z$ is the complex impedance of the shunting circuit; $C_{p}=\varepsilon_{33}^{T} A_{s}\left(h_{p}\right)^{-1}$ is the inherent capacitance of the piezoelectric patch; $A_{s}$ is the electrode area; and $h_{p}$ is the patch thickness.

Then the inherent elastic modules of the patch can be derived by Eq. (2) as

$$
E_{p}=\frac{h_{p}\left(1+s Z C_{p}\right)}{h_{p} s_{11}^{E}\left(1+s Z C_{p}\right)-s Z d_{31}^{2} A_{s}}
$$

As shown in Fig. 1(a), the negative capacitance circuit is used in the active control system to change the elastic modulus, in which the capacitance $C_{p}$, compensation resistance $R_{0}$, operational amplifier, fixed resistances $R_{1}$ and sliding rheostat $R_{2}$ are included. In the experiment, the operational amplifier LM324N is applied and the complex impedance $Z$ can be defined as

$$
Z=\frac{1}{-\alpha C_{p} s}
$$

where $\alpha=\left(R_{2} \times C\right) /\left(\mathrm{R}_{1} \times C_{p}\right)$.

Therefore, we can tune the value of $\alpha$ to change the inherent elastic modulus of piezoelectric patches and perform as the active control action on the elastic wave.

Numerical simulation. The periodic structure consists of a thin resin plate with $5 \mathrm{~mm}$ thickness and the periodic arrangement of surface-bonded hollow cylinders. The external diameter of the hollow cylinder is $34 \mathrm{~mm}$ and internal diameter is $26 \mathrm{~mm}$, as well as the height of the hollow cylinder is $30 \mathrm{~mm}$. These cylinders are arranged as the square lattice with the constant $40 \mathrm{~mm}$. As shown in Fig. 1(b), a T-shaped waveguide is designed by a $13 \times 13$ lattice and removing some cylinders. The horizontal channel is bonded by the circular P-4 piezoelectric 


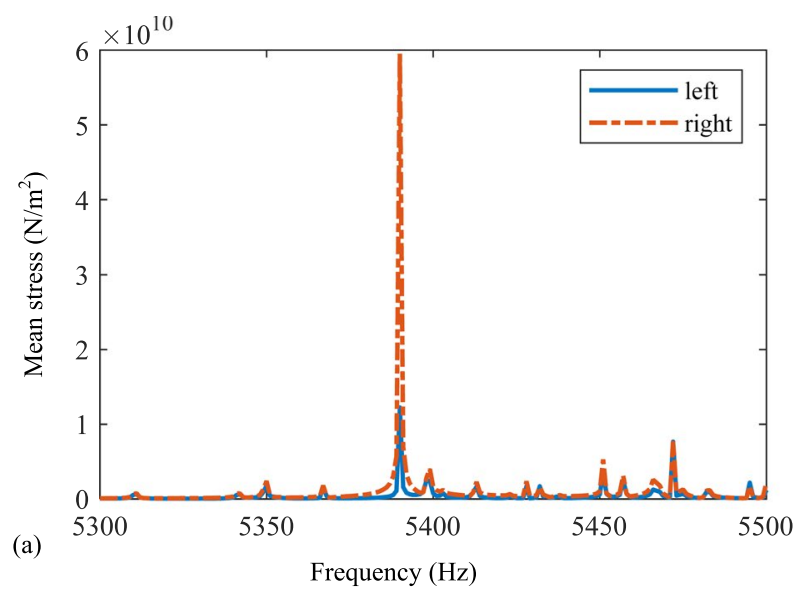

(b)

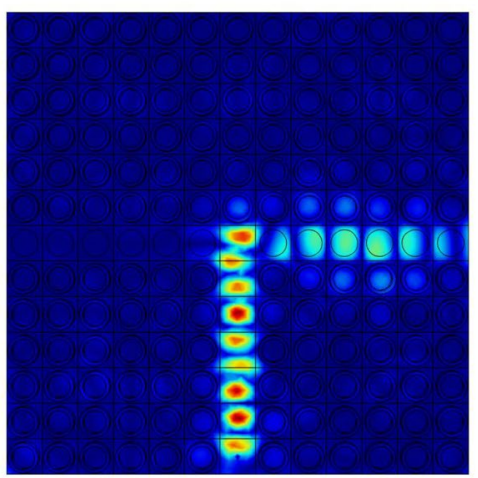

Figure 3. The switchable waveguide when the negative capacitance circuits are connected by the right side of the horizontal channel: (a) the mean stress of left and right from $5300 \mathrm{~Hz}$ to $5500 \mathrm{~Hz}$, and (b) the wave mode at $5390 \mathrm{~Hz}$.

\begin{tabular}{|l|l|l|l|l|l|}
\hline Material & $\begin{array}{l}\text { Mass } \\
\text { density } \rho \\
\left(\mathbf{k g} / \mathbf{m}^{3}\right)\end{array}$ & $\begin{array}{l}\text { Young's } \\
\text { modulus } E(\mathbf{N} / \\
\left.\mathbf{m}^{2}\right)\end{array}$ & $\begin{array}{l}\text { Compliance } \\
\text { coefficient } S_{11}^{E} \\
\left(\mathbf{m}^{3} \mathbf{N}^{-1}\right)\end{array}$ & $\begin{array}{l}\text { Piezoelectric } \\
\text { strain coefficient } \\
\mathbf{d} 31\left(\mathbf{C m}^{-2}\right)\end{array}$ & $\begin{array}{l}\text { Permittivity } \varepsilon_{33}^{T} \\
\left(\mathbf{F m}^{-1}\right)\end{array}$ \\
\hline p-4 & 7450 & $8.83 \times 10^{10}$ & $1.2 \times 10^{-11}$ & $-1 \times 10^{-10}$ & $1.2 \times^{-8}$ \\
\hline
\end{tabular}

Table 1. The material parameters of the piezoelectric patch.

patches with the diameter being $30 \mathrm{~mm}$ and the thickness being $1 \mathrm{~mm}$. As shown in Fig. 1(c), each patch is connected with a negative capacitance circuit to behave as the active control load.

The structural optimization by the optimization method, such as the genetic algorithm, is not performed. During the preparation of this work, several different kinds of the periodic structures are compared. Such as cuboid columns, solid and hollow cylinders are arranged as the square lattice and we find that the periodical structure with hollow cylinders can show the satisfied result. Then different structural parameters are considered to improve the model. We know that it's not the periodic structure with the best effect. It should be noted that in this work, the active control can show more importance.

The propagation properties of the elastic waves in the periodic structure are simulated by the finite element software COMSOL. As shown in Fig. 1(b), the flexural displacement is denoted by the red point to excite the elastic wave. Piezoelectric patches are attached to the left and right sides of the wave guide to generate the waveguide structure, which leads to band gap characteristics for both the right and left sides. We know that the elastic waves at some special frequencies will be localized near the defects of phononic crystals and metamaterials. This is the purpose to design this structure and the responses corresponding to the frequency from 0 to $6000 \mathrm{~Hz}$ are simulated by the finite element software. It can be found that the elastic wave is localized in the waveguide at $5390 \mathrm{~Hz}$. A notable process is changing the propagation direction of the elastic wave by the external active control system. Then the negative capacitance circuits are connected by the right side of the horizontal channel and the parameter $\alpha=0.9$ is applied.

As shown in Fig. 2(a), the mean stress of the left and right sides are calculated as the elastic wave frequency changes from $5300 \mathrm{~Hz}$ to $5500 \mathrm{~Hz}$. The solid and dotted lines represent the mean stresses on the left and right 

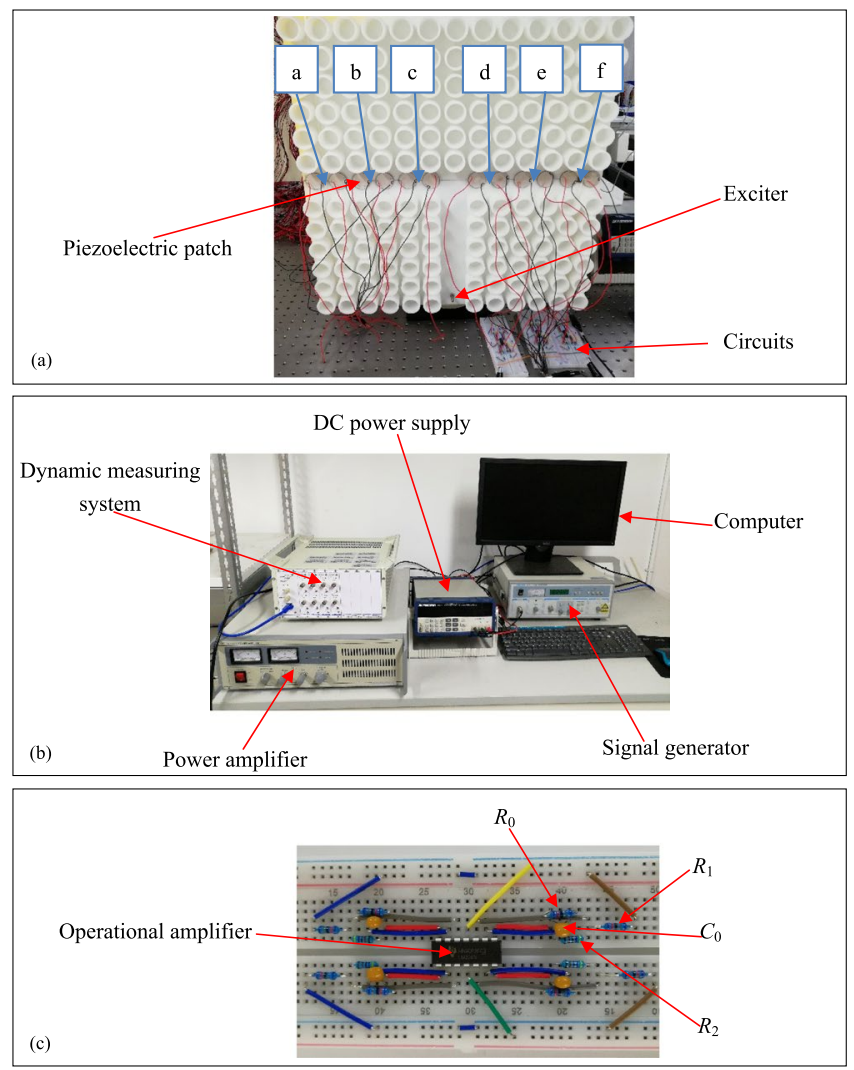

Figure 4. Experimental setup: (a) The switchable waveguide with the active control system, (b) the testing system and (c) the negative capacitance circuit.

\begin{tabular}{|l|l|l|l|l|l|}
\hline $\boldsymbol{C}(\boldsymbol{p} F)$ & $C_{p}(p F)$ & $\boldsymbol{R}_{2}(\mathbf{k} \Omega)$ & $\begin{array}{l}\boldsymbol{R}_{1} \\
(\mathbf{k} \Omega)\end{array}$ & $\begin{array}{l}\mathbf{R}_{0} \\
(\mathbf{k} \Omega)\end{array}$ & $\begin{array}{l}\text { Operational } \\
\text { amplifier }\end{array}$ \\
\hline 9.684 & 8.155 & 51.54 & 68 & 2000 & LM324N \\
\hline
\end{tabular}

Table 2. The parameters of the negative capacitance circuit.

sides. We can see that when the wave frequency is $5390 \mathrm{~Hz}$, the mean stress on the left side is much larger than that on the right side. According to the corresponding wave mode in Fig. 2(b), we can obviously find that the propagation of the elastic wave turns left when it arrives at the bifurcation point, and the elastic wave cannot propagate along the right side. It's mainly because that the negative capacitance circuits change the equivalent elastic modules of the left-sided waveguide and the band structure will be changed. Then the line defect is formed, which can behave as the waveguide. Then the direction of the elastic wave will change and it propagates along the left side at the frequency $5390 \mathrm{~Hz}$.

In order to compare these results, the negative capacitance circuits are applied on the left side of the horizontal channel. The mean stress of the left and right sides with the same frequency range from $5300 \mathrm{~Hz}$ to $5500 \mathrm{~Hz}$ are simulated, and the results are shown in Fig. 3(a). The solid and dotted lines denote the mean stresses on the left and right sides. Then the similar conclusion can be obtained that the mean stress on the right side is much larger than that on the right one. From the wave mode distribution in Fig. 3(b), we can see that the elastic wave turns right when it arrives at the bifurcation, and cannot propagate along the left side when the frequency is $5390 \mathrm{~Hz}$. Due to the line defect appears at this frequency, which can bound the propagation of the elastic wave. So the elastic wave will change the direction and propagate along the right side. Then the active control of the elastic wave metamaterials on the switchable properties is achieved.

Experimental setup. To demonstrate the validity of the numerical simulations, we carry out the active control experiments on the waveguide structure, in which the P-4 piezoelectric patches are boned at the line defect. The waveguide structure is fabricated by the $3 \mathrm{D}$ printing technology. The Young's modulus $E=2.5 \mathrm{GPa}$, the density $\rho=1300 \mathrm{~kg} / \mathrm{m}^{3}$ and the Poisson's ratio $\nu=0.41$. The material parameters of the $\mathrm{P}-4$ piezoelectric patch are shown in Table 1.

The experimental setup is illustrated in Fig. 4. The waveguide structure is connected with the negative capacitance circuits. The elastic wave exciter and the collected points a-f are shown in Fig. 4(a). Figure 4(b) illustrates the experimental testing system, in which the signal is generated and the responses are received and saved in the 

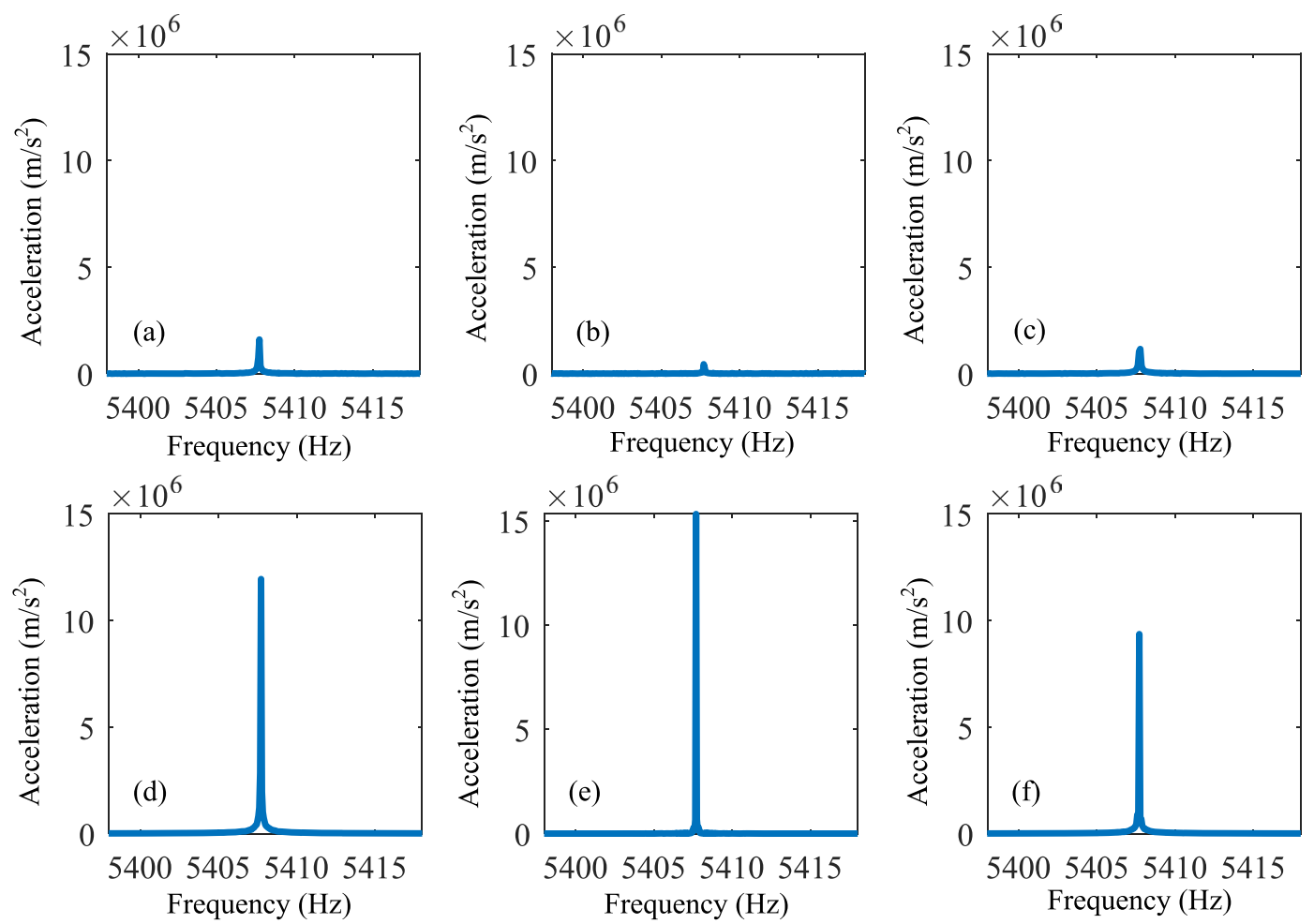

Figure 5. Responses of points a-f at $5408 \mathrm{~Hz}$ when the left side of the horizontal channel is exerted by the active control system.
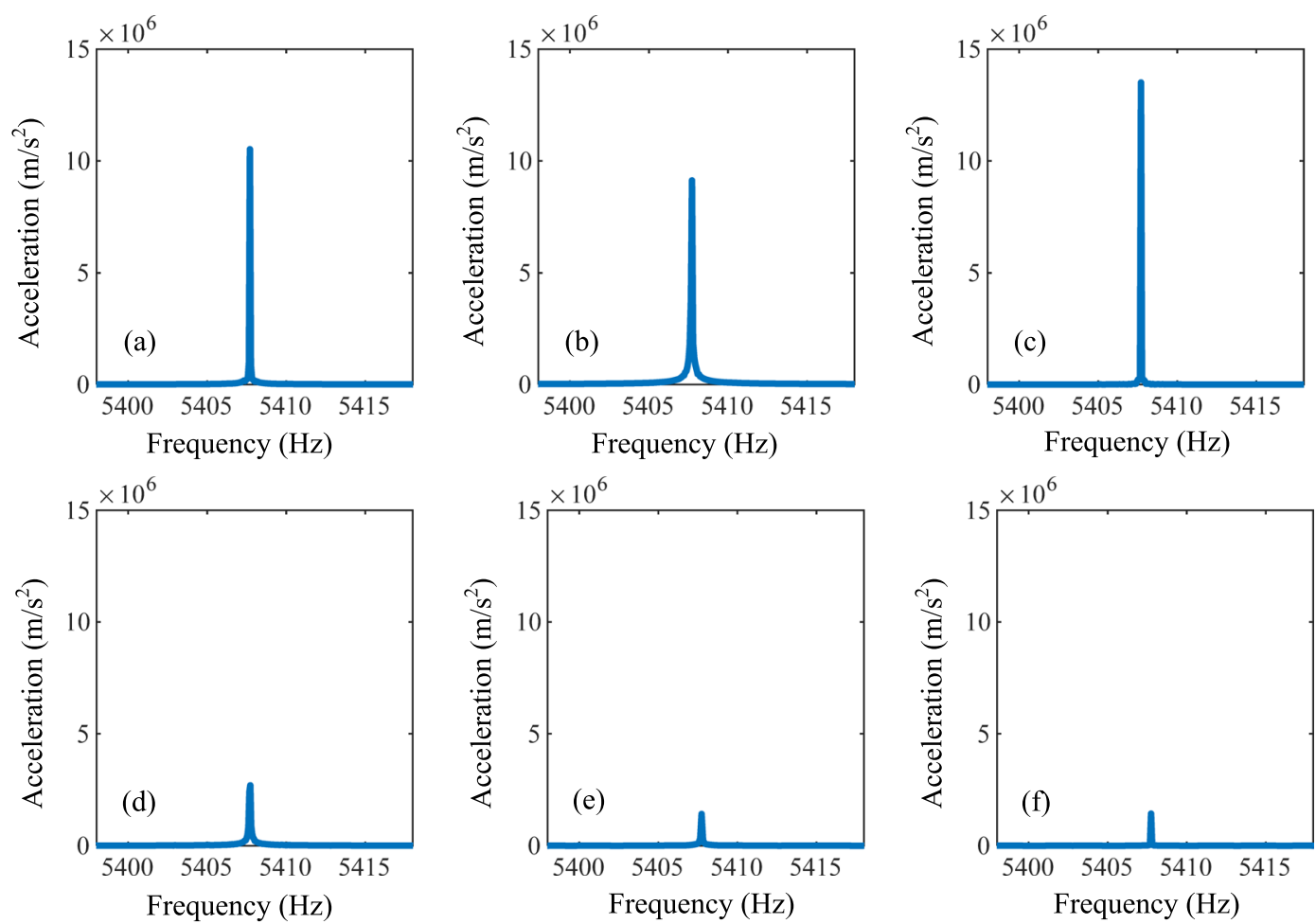

Figure 6. Responses of points a-f at frequency at $5408 \mathrm{~Hz}$ when the right side of the horizontal channel is exerted by the active control system. 
computer. As shown in Fig. 4(c), the DC power supply depends on the operational amplifier, which is an important device in the negative capacitance circuit. The explicit description of the circuit is listed in Table 2.

Figure 5 and 6 show the experimental results, in which the subsets (a-f) correspond to the responses of the points a-f. Considering the connecting circuits on the left side of the horizontal channel, we can see that the responses of the points $\mathrm{a}-\mathrm{c}$ are much smaller than those of the points $\mathrm{d}-\mathrm{f}$ at $5408 \mathrm{~Hz}$. It means that at this frequency, most energy of the elastic wave propagates along the right side. Moreover, if the negative capacitance circuits are connected by the right side of the horizontal channel, we can find that the responses of the points a-c are much larger than those of the points $\mathrm{d}-\mathrm{f}$ at $5408 \mathrm{~Hz}$. It can be concluded that the elastic wave can hardly transmit along the right side but propagate along the left side very well.

We know that the experimental testing system cannot present the displacement distribution field like the numerical results. It can test the acceleration signal at a certain point. So we set three receiving points for the acceleration signal on the left or right side to get the information about the wave propagation. When the acceleration signals of the three points on the left are much higher than those on the right, we can assume that the elastic wave changes the direction and propagates along the left of the waveguide. On the other hand, similar behaviors can also be derived if the wave propagates along the right side. These acceleration responses are shown in Figs 5 and 6 . Then, the response for the frequency at $5408 \mathrm{~Hz}$ appears a satisfied result which is close to the numerical simulation for the frequency being $5390 \mathrm{~Hz}$. As a result, the active control of the wave propagation can be supported well by the experiments.

\section{Conclusions}

Previous investigations on the elastic waveguides are mainly concentrated on the propagation of elastic waves only along a single direction. In this work, the switchable waveguide of the elastic wave metamaterials is achieved by the active control action. In order to control the material parameters, the switchable waveguide is connected with the piezoelectric patches which are attached by the negative capacitance circuits. Furthermore, the experimental model is fabricated by the 3D printing technology and bonded by the active control system. The validity of the numerical simulation is supported by the experiment. This study is expected to be helpful to design the elastic wave metamaterials with the active control action.

Received: 12 June 2019; Accepted: 18 October 2019;

Published online: 07 November 2019

\section{References}

1. Mehaney, A. Phononic crystal as a neutron detector. Ultrasonics 93, 37-42 (2019).

2. Elmadih, W. et al. Three- dimensional resonating metamaterials for low-frequency vibration attenuation. Scientific Reports 9 , 11503 (2019).

3. Krodel, S. \& Delpero, T. 3D auxetic microlattices with independently controllable acoustic band gaps and quasi-static elastic moduli. Advanced Engineering Materials 4, 357-363 (2014).

4. Ahmed, R. U. \& Banerjee, S. Low frequency energy scavenging using sub-wave length scale acousto-elastic metamaterial. AIP Advances 4, 117114 (2014).

5. Han, J. N. \& Tang, S. Realization of complex curved waveguide based on local resonant 3D metamaterial. AIP Advances 8, 125327 (2018).

6. Warmuth, F., Wormser, M. \& Korner, C. Single phase 3D phononic band gap material. Scientific Reports 7, 3843 (2018).

7. Hyun, J., Choi, W., Wang, S., Park, C. S. \& Kim, M. Systematic realization of double-zero-index phononic crystals with hard inclusions. Scientific Reports 8, 7288 (2018).

8. Surjadi, J. U. et al. Mechanical metamaterials and their engineering applications. Advanced Engineering Materials 21, 1800864 (2019).

9. Li, S. B. et al. Designing a broad locally-resonant bandgap in a phononic crystals. Physics Letters A 382, 1371-1377 (2019).

10. Beli, D., Fabro, A. T., Ruzzene, M. \& Arruda, J. R. F. Wave attenuation and trapping in 3D printinged cantilever-in-mass metamaterials with spatially correlated variability. Scientific Reports 9, 5617 (2019).

11. Yudistira, D., Boes, A., Djafari-Rouhani, B. \& Pennec, Y. J. R. Monolithic phononic crystals with a surface acoustic band gap from surface phonon-polariton coupling. Physical Review Letters 113, 215503 (2014).

12. Dong, H. W., Wang, Y. S. \& Zhang, C. Z. Topology optimization of chiral phoxonic crystals with simultaneously large phononic and photonic bandgaps. IEEE Photonics Journal 9, 19430655 (2017).

13. V Zhu, R., Liu, X. N., Hu, G. K., Sun, C. T. \& Huang, G. L. Negative refraction of elastic waves at the deep-subwavelength scale in a single-phase metamaterial. Nature Communications 5, 5510 (2014).

14. Croenne, C., Morvan, B., Vasseur, J., Dubus, B. \& Hladky-Hennion, A. Analysis of elastic waves transmitted through a 2-D phononic crystal exhibiting negative refraction. IEEE Transactions on Ultrasonics Ferroelectrics and Frequency Control 58, 178-186 (2011).

15. Philippe, F. D., Murray, T. W. \& Prada, C. Focusing on plates: controlling guided waves using negative refraction. Scientific Reports 5, $11112(2015)$.

16. Sukhovich, A., Jing, L. \& Page, J. H. Negative refraction and focusing of ultrasound in two-dimensional phononic crystals. Physical Review B 77, 014301 (2008).

17. Torrent, D. \& Sánchez-Dehesa, J. Acoustic cloaking in two dimensions: a feasible approach. New Journal of Physics 10, 063015 (2008).

18. Zhang, S., Xia, C. \& Fang, N. Broadband acoustic cloak for ultrasound waves. Physical Review Letters 106, 024301 (2011).

19. Farhat, M. et al. A homogenization route towards square cylindrical acoustic cloaks. New Journal of Physics 10, 115030 (2008)

20. Herrmann, H., Schaefer, K. \& Sohler, W. Polarization independent, integrated optical, acoustically tunable wavelength filters/ switches with tapered acoustical directional coupler. IEEE Photonics Technology Letters 6, 1335-1337 (1994).

21. Lucklum, R., Zubtsov, M. \& Ke, M. Liquid sensor utilizing a regular phononic crystal with normal incidence of sound. IEEE Transactions on Ultrasonics Ferroelectrics and Frequency Control 59, 463-471 (2012).

22. Nishizawa, J. et al. THz generation from gap rod-type waveguides. IEEE Photonics Technology Letters 19, 143-145 (2007).

23. Rostami-Dogolsara, B., Moravvej-Farshi, M. \& Nazari, F. Designing switchable phononic crystal-based acoustic demultiplexer. IEEE Transactions on Ultrasonics Ferroelectrics and Frequency Control 63, 1468-1473 (2016).

24. Zhang, X., Liu, Z., Liu, Y. \& Wu, F. Defect states in $2 \mathrm{~d}$ acoustic band-gap materials with bend-shaped linear defects. Solid State Communications 130, 67-71 (2004). 
25. Wang, Y. F., Wang, T. T., Wang, Y. S. \& Laude, V. Reconfigurable phononic crystal circuits formed by coupled acoustoelastic resonators. Physical Review Applied 8, 014006 (2017).

26. Wang, Y. F., Wang, T. T., Liu, J. P., Wang, Y. S. \& Laude, V. Guiding and splitting Lamb waves in coupled-resonator elastic waveguides. Composite Structures 206, 588-593 (2018).

27. Yao, Z. J., Yu, G. L., Wang, Y. S., Shi, Z. F. \& Li, J. B. Propagation of flexural waves in phononic crystal thin plates with linear defects. Journal of Zhejiang University-Science A 11, 827-834 (2010).

28. Zhang, S. Z. \& Gao, Y. W. Gap evolution of Lamb wave propagation in magneto-elastic phononic plates with pillars and holes by modulating magnetic field and stress loadings. Journal of Applied Physics 124, 244102 (2018).

29. Wen, J. H., Shen, H. J., Yu, D. L. \& Wen, X. S. Exploration of amphoteric and negative refraction imaging of acoustic sources via active metamaterials. Physics Letters A 377, 2199-2206 (2013).

30. Chaunsali, R., Li, F. \& Yang, J. Stress wave isolation by purely mechanical topological phononic crystals. Scientific Reports 6, 30662 (2016).

31. Lossouarn, B., Deu, J. F. \& Aucejo, M. Multimodal vibration damping of a beam with a periodic array of piezoelectric patches connected to a passive electrical network. Smart Materials and Structures 24, 115037 (2015).

32. Zhou, W. J. et al. Actively tunable transverse waves in soft membrane-type acoustic metamaterials. Journal of Applied Physics 123, 165304 (2018)

33. Tateo, F., Collet, M., Quissen, M., Cunefare, K. \& Abbe, P. Experimental characterization of a bi-dimensional array of negative capacitance piezo-patches for vibroacoustic control. Journal of Intelligent Material Systems and Structures 26, 952-964 (2015).

34. Casadei, F., Beck, B., Cunefare, K. A. \& Ruzzene, M. Vibration control of plates through hybrid configurations of periodic piezoelectric shunts. Journal of Intelligent Material Systems and Structures 23, 1169-1177 (2012).

35. Chen, Y. Y., Zhu, R., Barnhart, M. V. \& Huang, G. L. Enhanced flexural wave sensing by adaptive gradient-index metamaterials. Scientific Reports 6, 35048 (2016).

36. Yuan, S.Q., Chua, C.K., Zhou, K. 3D-printed mechanical metamaterials with high energy absorption. Advanced Materials Technologies, 1800419 (2018).

37. Hagood, N. W. \& Flotow, A. V. Damping of structural vibrations with piezoelectric materials and passive electrical networks. Journal of Sound and Vibration 146, 243-268 (1991).

38. Wang, G. \& Chen, S. Large low-frequency vibration attenuation induced by arrays of piezoelectric patches shunted with amplifier-resonator feedback circuits. Smart Materials and Structures 25, 015004 (2015).

\section{Acknowledgements}

The authors acknowledge the supports by the National Natural Science Foundation of China (Grant Nos. 11772039 and 11532001), the Joint Sino-German Research Project (Grant No. GZ 1355) and the German Research Foundation (DFG, Grant No. ZH 15/27-1) for this research work.

Author contributions

Guan-Hua Li performed the numerical simulation and experiment. Yi-Ze Wang discussed the results and developed the experimental model. Yue-Sheng Wang presented the helpful suggestions about the underlying mechanism and participated in the revision of the manuscript. All authors contributed to the writing and editing of the manuscript.

\section{Competing interests}

The authors declare no competing interests.

\section{Additional information}

Correspondence and requests for materials should be addressed to Y.-Z.W.

Reprints and permissions information is available at www.nature.com/reprints.

Publisher's note Springer Nature remains neutral with regard to jurisdictional claims in published maps and institutional affiliations.

Open Access This article is licensed under a Creative Commons Attribution 4.0 International License, which permits use, sharing, adaptation, distribution and reproduction in any medium or format, as long as you give appropriate credit to the original author(s) and the source, provide a link to the Creative Commons license, and indicate if changes were made. The images or other third party material in this article are included in the article's Creative Commons license, unless indicated otherwise in a credit line to the material. If material is not included in the article's Creative Commons license and your intended use is not permitted by statutory regulation or exceeds the permitted use, you will need to obtain permission directly from the copyright holder. To view a copy of this license, visit http://creativecommons.org/licenses/by/4.0/.

(c) The Author(s) 2019 